\title{
Lossless digital filter overflow oscillations; approximation of invariant fractals.
}

\author{
Peter Ashwin, W. Chambers ${ }^{\dagger}$ and G. Petkov ${ }^{\dagger}$ \\ January 17, 1997
}

\begin{abstract}
We investigate second order lossless digital filters with two's complement overflow. We numerically approximate the fractal set $D$ of points that iterate arbitrarily close to the discontinuity. For the case of eigenvalues of the associated linear map of the form $e^{i \theta}$ with $\theta / \pi \notin \mathbf{Q}$ we present evidence that $D$ has positive two dimensional Lebesgue measure. For $\theta / \pi \in \mathbf{Q}$ we confirm that $D$ has Lebesgue measure zero. As a by-product we get estimates of the exterior dimension of $D$. These results imply that if such filters are realised using finite-precision arithmetic then they will have a sizeable fraction of orbits that are periodic with high period overflows.
\end{abstract}

\section{Introduction}

It has been recognised for several years that lossless digital filter overflow oscillations with two's complement overflows can exhibit very complex behaviour; see [Chua \& Lin, 1988], [Davies 1995] and [Kocarev et al., 1996] for recent work on this problem. However, there are still several questions for this system that remain unanswered; in particular, what is the nature of fractal on which "chaotic" behaviour is seen? What is the nature of the "chaotic" dynamics on it? How does this depend on parameters of the system? We address the first of these questions in this paper.

We define $I=[-1,1)^{2}$ and note that a second order digital filter with two's complement overflow (in a continuum approximation) can be written as a map $f: I \rightarrow I$ where

$$
f(x, y)=(y, g(b x+a y))
$$

with $(a, b) \in \mathbf{R}^{2}$ the parameters of the map. The overflow is governed by the function $g$ defined by

$$
g(x)=x \quad(-1 \leq x<1), \quad g(x+2)=g(x) \quad(x \in \mathbf{R}) .
$$

${ }^{*}$ Dept. of Mathematical and Computing Sciences, University of Surrey, Guildford GU2 5XH, UK. Email: P.Ashwin@mcs.surrey.ac.uk

${ }^{\dagger}$ Dept. of Electronic and Electrical Engineering, Kings College, University of London, Strand, London WC2 2LS, UK. 
The actual value taken by $g$ at its discontinuities $x \in 1+2 \mathbf{Z}$ is in fact unimportant for what follows. If $g(b x+a y)>1$ we say that point causes overflow; if $g(b x+a y)<-1$ we say it causes underflow.

We restrict to the lossess case $b=-1$ and $|a|<2$ and define $\theta \in(0, \pi)$ such that $a=2 \cos \theta$; the eigenvalues of (1) with $g(x)=x$ are precisely $e^{ \pm i \theta}$. In this lossless case the map preserves two dimensional Lebesgue measure which we write as $\ell()$ and normalise such that $\ell(I)=1$. We give $I$ the topology of the torus; this can be done such that there is a single line of discontinuity at

$$
\mathcal{D}=\{(-1, y): y \in[-1,1)\}
$$

We consider the set of all pre- and post-images of this set; namely

$$
\mathcal{D}^{ \pm}=\bigcup_{n \in \mathbf{Z}} f^{n}(\mathcal{D})
$$

where the image of the discontinuity is the set of all limit points of images of points that tend to the discontinuity. This set (called $I_{\gamma}$ in [Kocarev et al., 1996]) has two-dimensional Lebesgue measure zero because it is a countable union of onedimensional sets. We define

$$
\tilde{I}=I \backslash \mathcal{D}^{ \pm}
$$

which differs from $I$ on a set of measure zero and consider the dynamics here.

We can partition this set into two disjoint sets as follows:

$$
D=\tilde{I} \cap \overline{\mathcal{D}^{ \pm}}
$$

the closure of the set of (pre)images of the discontinuity, and

$$
D^{c}=\tilde{I} \backslash D
$$

As noted in [Ashwin, 1996], all periodic points in $\tilde{I}$ must be contained in $D^{c}$. Moreover $D^{c}$ consists of a disjoint union of convex polygons that are mapped to themselves by some iterate of $f$. We call one of these polygons a periodic island. Each periodic island can be uniquely distinguished by its overflow sequence; this is an infinite sequence of the symbols $\{-,+, 0\}$ corresponding to whether the $n$th iterate of a point in the island causes underflow, overflow or neither, respectively. We say a point has periodic overflow if its overflow sequence is periodic. Now define

$$
\Phi=\ell(D)
$$

(a function of $a$ ) be the two dimensional Lebesgue measure of this set (this is measurable as it is a countable union of 1 -d sets). We approximate $\Phi(a) \in[0,1]$ by discretising the phase space. In [Ashwin, 1996] it was conjectured that $\Phi(a)>0$ for almost all values of $a \in(-2,2)$; by contrast, [Kocarev et al., 1996] conjecture that $\Phi(a)=0$ for all $a$. 


\section{Finite grid approximation}

We consider $I_{(n)}$, a $2^{n} \times 2^{n}$ discretisation of the phase space into $2^{2 n}$ cells. We approximate the discontinuity $\mathcal{D}$ by $\mathcal{D}_{(n)}$, the set of points that lie on the left-hand edge of $I_{(n)}$.

An approximation of $D$ was computed in the following way; initial points were chosen in the centers of boxes in $I_{(n)}$ bordering one side of the discontinuity. These points were iterated using double precision arithmetic and each cell hit during the orbit was "coloured in". When the trajectory returned to its initial cell it was assumed to have closed. Due to Poincaré recurrence, almost all initial points will return to any neighbourhood of their initial points. We therefore compute $D_{(n)}$; the set of all "coloured in" boxes as an approximation to $D$ and define

$$
\Phi_{n}(a)=\frac{N\left(D_{(n)}\right)}{2^{2 n}}
$$

where $N(S)$ is the number of cells in $S$. The set $D_{(n)}$ is an approximation of $B_{2^{-n}}(D)$ where $B_{\epsilon}(D)$ is the $\epsilon$-parallel body of $D$, i.e. the set of all points within $\epsilon$ of some point in $D$.

\subsection{Approximation of $\ell(D)$}

We conjecture that $\Phi(a)$ has positive measure for most values of $a$ and so $D$ is a fat fractal [Farmer, 1985]. If $\ell(D)>0$ then the Hausdorff dimension of $d$ is two but the boundaries may be very convoluted; as a measure of this we use the exterior dimension $\left(d_{e x}\right)$ to further characterise such fat fractal sets.

The exterior dimension [Grebogi et al., 1985] of a two dimensional set $D$ is defined to be $d=d_{e x}$ such that the scaling

$$
\ell\left(B_{\epsilon}(D)\right)=\ell(D)+A \epsilon^{2-d}+o\left(\epsilon^{2-d}\right)
$$

with $A>0$ holds as $\epsilon \rightarrow 0$. This is related to the uncertainty exponent $\alpha$ [Farmer, 1985] of a fat fractal by

$$
2-d_{e x}=\alpha
$$

and so these two quantities are equivalent. If $\ell(D)=0$ then $(2)$ is a formula for the Minkowski dimension $d$ (or box-counting dimension) of $D$ [Falconer, 1990]; this is an upper bound for the Hausdorff dimension of $D$, and for many sets the two dimensions are equal.

From (2) we expect the asymptotic behaviour

$$
\Phi_{n}(a)=\Phi_{\infty}(a)+A \exp ^{-n\left(2-d_{e x}\right) \ln 2}
$$

as $n \rightarrow \infty$ and so fitting a curve $A \exp (n B)+C$ to calculated values for $\Phi_{n}$ gives us both $\Phi_{\infty}=C$ and $d_{e x}=2+B / \ln 2$.

Examples of $D_{(n)}$ and $\Phi_{n}$ with fitted curve are shown in Figure 1, where of $\Phi_{n}(1.5)$ $\left(\theta=41.409622^{\circ}\right)$ has been computed for $n$ from 2 up to 12 . The fitted curves shows that the values obtained are consistent with $\Phi(1.5) \sim 0.121>0$. Figure 2 shows 
the case $\Phi_{n}(\sqrt{2})\left(\theta=45^{\circ}\right)$; this indicates that $\Phi(\sqrt{2})=0$ as expected. As a second example of a rational angle, Figure 3 shows the scaling of $\Phi_{n}$ for $a=0.44504=$ $2 \cos 3 \pi / 7$. This also shows good convergence to zero Lebesgue measure.

The data for scans of $a$ from 0 to 2 in steps of 0.0005 are shown in Figure 4; $n=6, n=8$ and $n=10$ are shown. Fitting these three values to the unique curve of the form

$$
A \exp (B n)+C
$$

gives extrapolations to $\Phi_{\infty}$ and $d_{e x}$. Due however to the behaviour of $\Phi_{n}$ not being necessarily asymptotic in this region, there can be spurious predictions of $\Phi_{\infty}<0$, $\Phi_{\infty}>\Phi_{n}, d_{e x}<1$ or $d_{e x}>2$. These four cases have been suppressed. On the remaining points, there is evidence that the curves limit to a curve that is non-zero almost everywhere, with the presence of sharp 'dips' near $a=2 \cos \theta$ with $\theta / \pi \in \mathbf{Q}$ indicative of $\Phi(a)=0$ at this dense set of values of $a$. In Figure 5 we show the extrapolation of this data to give an approximation of the exterior dimension of $D$ as a function of $a$.

\subsection{Approximation of $\ell\left(D^{c}\right)$}

One can approximate the measure of $D$ by approximating the measure of the complement $D^{c}$ and noting that $\ell(D)+\ell\left(D^{c}\right)=1$. The area taken up by periodic islands in $D^{c}$ with simple overflow sequences has been calculated by [Davies, 1995] and [Davies \& Petkov, 1995]. They have analytical formulae for the proportion of phase space that is taken up by some of the simplest overflow sequences. This does not cover all periodic islands, but it does seem to cover the majority of the area for a wide range of parameters $a$.

Figure 6 shows the area in $I$ taken up by periodic islands corresponding to simple overflow sequences as a function of $a$; each colour corresponds to a different overflow sequence and was calculated by [Davies \& Petkov, 1995]. For comparison, $\Phi(a)$ calculated with $n=10$ (see Figure 4) is shown on the same axes. This curve lies wholly below the area of the islands as calculated by [Davies \& Petkov, 1995] and so the two approximations are consistent with $\ell(D)+\ell\left(D^{c}\right)=1$.

It is interesting to note that near $a=1$ the two curves approach very close to each other. This indicates that for $a$ limiting to one, the total area of all except two of the islands tends to zero. The two islands left correspond to no overflow and period two overflow.

\subsection{Hausdorff dimension}

For $a=\sqrt{2}$, one can explicitly construct $D$ by virtue of its self-similarity shown schematically in Figure 7. We can use three similarity transformations as in Figure 7 with similarity factors $c=3-2 \sqrt{2}$ to generate the fractal pattern. Using a result in [Falconer, 1990] one compute the Hausdorff dimension of $D$ to be $\nu$ such that

$$
3 c^{\nu}=1 \quad \text { i.e. } \quad \nu=-\frac{\ln 3}{\ln c}=1.37676
$$


By comparison, the Minkowski dimension approximated by fitting (3) is 1.3835 ; this agrees fairly well with the theoretical calculation of the Hausdorff dimension. Moreover, it gives credence to dimension predictions for other values of $a$.

\section{Discussion}

The numerical experiments presented here give support to the conjecture that $D$ has positive two-dimensional Lebesgue measure for $a=2 \cos \theta$ with $\theta / \pi$ irrational. We have not examined the dynamics of $f$ on $D$ in this paper, but note that although the Liapunov exponents of points in $D$ are defined and zero, this does not imply that dynamics in $D$ does not have, for example, sensitive dependence on initial conditions. Thus it may still be chaotic in a fairly strong sense. In particular, the definition of $D$ means that for any $(x, y) \in D$ the map is not conjugate to its linearisation on any neighbourhood of $(x, y)$ and so there are always nearby trajectories that will separate from that of $(x, y)$.

Some aspects of the behaviour of this map are similar to the behaviour of interval exchange maps defined as follows. Take an interval $[0,1]$, cut it into $n$ pieces of a given size and re-assemble to give $[0,1]$ again. One finds that generically one can split $[0,1]$ into a disjoint union of two sets, each of which is a union of intervals. In one set there are dense orbits, while in the other all orbits are periodic [Katok \& Hasselblatt, 1995]; these sets are analogous to the sets $D$ and $D^{c}$ discussed above.

\subsection{Finite precision calculations}

The computations in section 2 were performed using double precision numerics and grids with between $2^{4}$ and $2^{24}$ cells. If we perform $n$ - bit arithmetic on a phase space that is a $2^{n}$ by $2^{n}$ grid we can study the dynamics of finite precision implementations of the map (1).

The main effect of performing the calculations at finite precision appears to be to add noise (from rounding errors) to the double precision calculations. This means that typical trajectories diffuse more in the phase space and consequently more points come close to the discontinuity of (1).

We have numerically investigated this approximation and find the approximation of $D$ using $n$-bit arithmetic behaves similarly to $D_{(n)}$ (see Figure 8 for an example with $n=10$ and $a=\sqrt{2}$ ). The addition of the rounding error has the effect of causing trajectories to explore a 'halo' around the expected position of $D$ (compare this to Figure $2(\mathrm{~b})$ ). Figure 9 shows comparison of $\Phi_{n}$ calculated by double precision and by $n$-bit arithmetic for $a=1.5$ and $n=3, \cdots, 12$. The finite precision results can be seen to decay less regularly that the double precision results and the former show a consistently larger proportion of orbits coming near the discontinuity. 
Ashwin, P. [1996] "Non-smooth invariant circles in digital overflow oscillations," Proceedings of NDES96: fourth international workshop on Nonlinear Dynamics of Electronic Systems, Seville, Spain.

Chua, L.O. \& Lin, T. [1988] "Chaos in digital filters," IEEE Trans CAS 35 pp. 648-658.

Davies, A.C. [1995] "Nonlinear oscillations and chaos from digital filter overflow," Phil. Trans. R. Soc. Lond. A 353 pp. 85-99.

Davies, A.C. \& Petkov, G.P. [1995] "Overflow limit cycles from digital filters with complex eigenvalues within the unit circle," Proc. of NDES95: 3rd international workshop on Nonlinear Dynamics of Electronic Systems, Dublin, Ireland pp 45-48.

Falconer, K. [1990] Fractal Geometry. (John Wiley, Chichester).

Farmer, J.D. [1985] "Sensitive dependence on parameters in nonlinear dynamics," Phys. Rev. Lett. 55 pp. 351-354.

Grebogi, C., McDonald, S.W., Ott, E. \& Yorke, J.A. [1985] "Exterior dimensions of fat fractals," Phys. Lett. A 110 pp. 1-4.

Katok, A. \& Hasselblatt, B. [1995] Introduction to the modern theory of dynamical systems Encylopedia of Mathematics and its Applications; vol 54 (CUP, Cambridge).

Kocarev L., Wu, C.W. \& Chua, L.O. [1996] "Complex behaviour in Digital filters with overflow nonlinearity: analytical results," IEEE Trans CAS-II 43 pp. 234-246. 


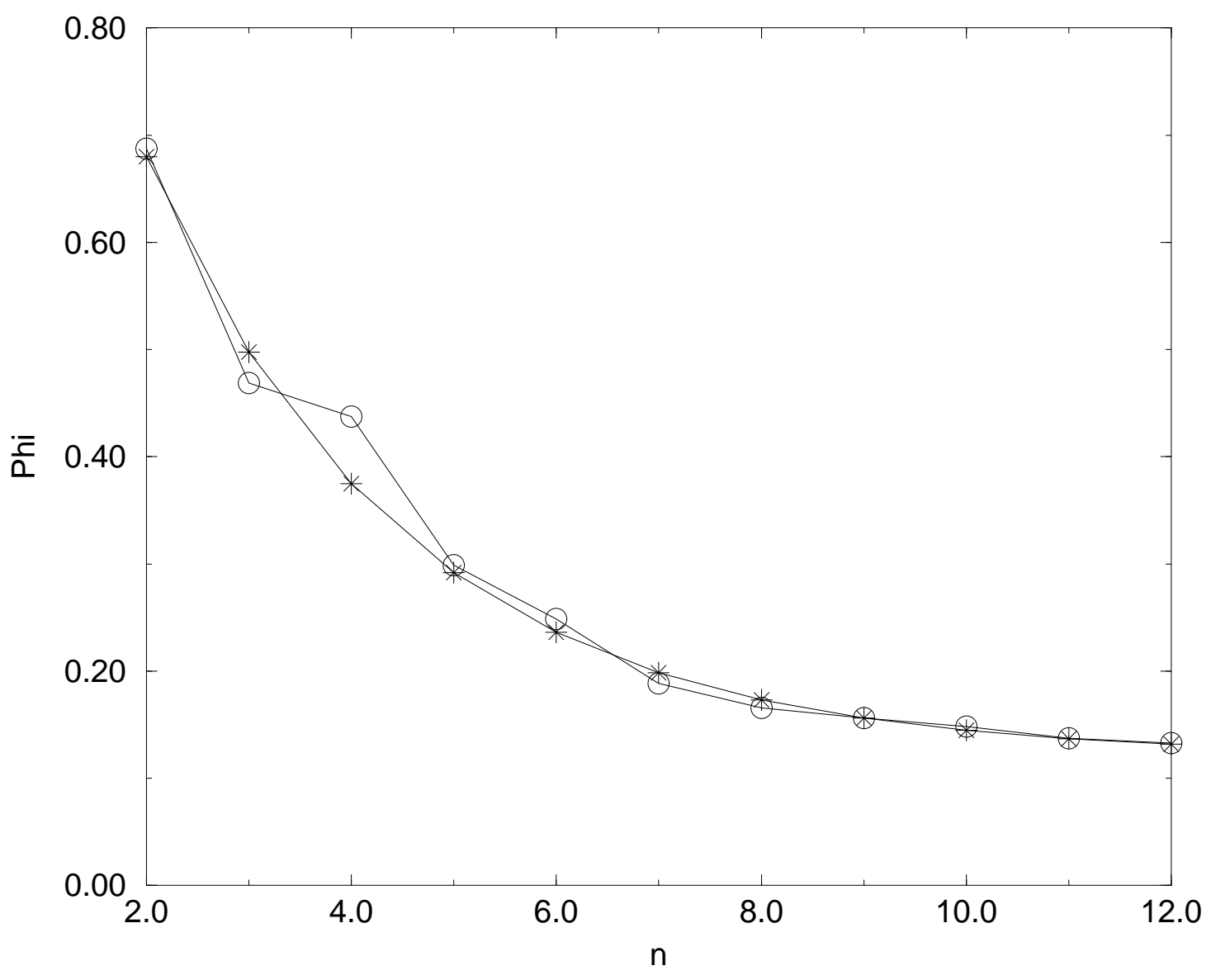

(a) 


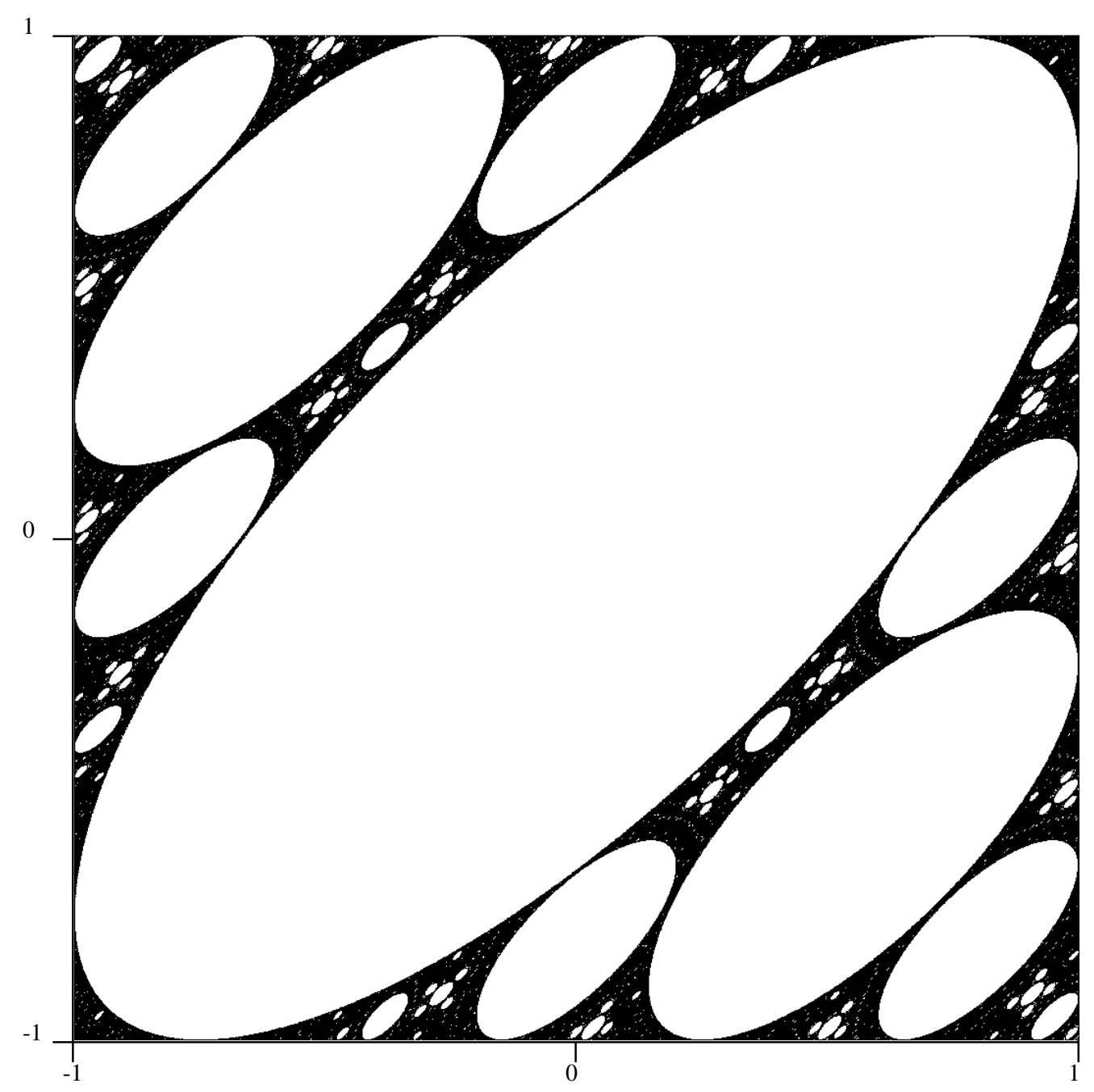

(b)

Figure 1: (a) The points marked by circles show $\Phi_{n}(1.5)$ as a function of $n=$ $2, \cdots, 12$. The stars show a fit to the function $0.121+1.2316 e^{-0.395 n}$, i.e. $\Phi_{\infty}(1.5)=$ 0.121 and $d_{e x}=1.57$. Such results are typical for $a=2 \cos \theta$ with $\theta / \pi \notin \mathbf{Q}$. (b) Approximation of $D$ for $n=10$ in the plane $(x, y) \in[-1,1)^{2}$. 


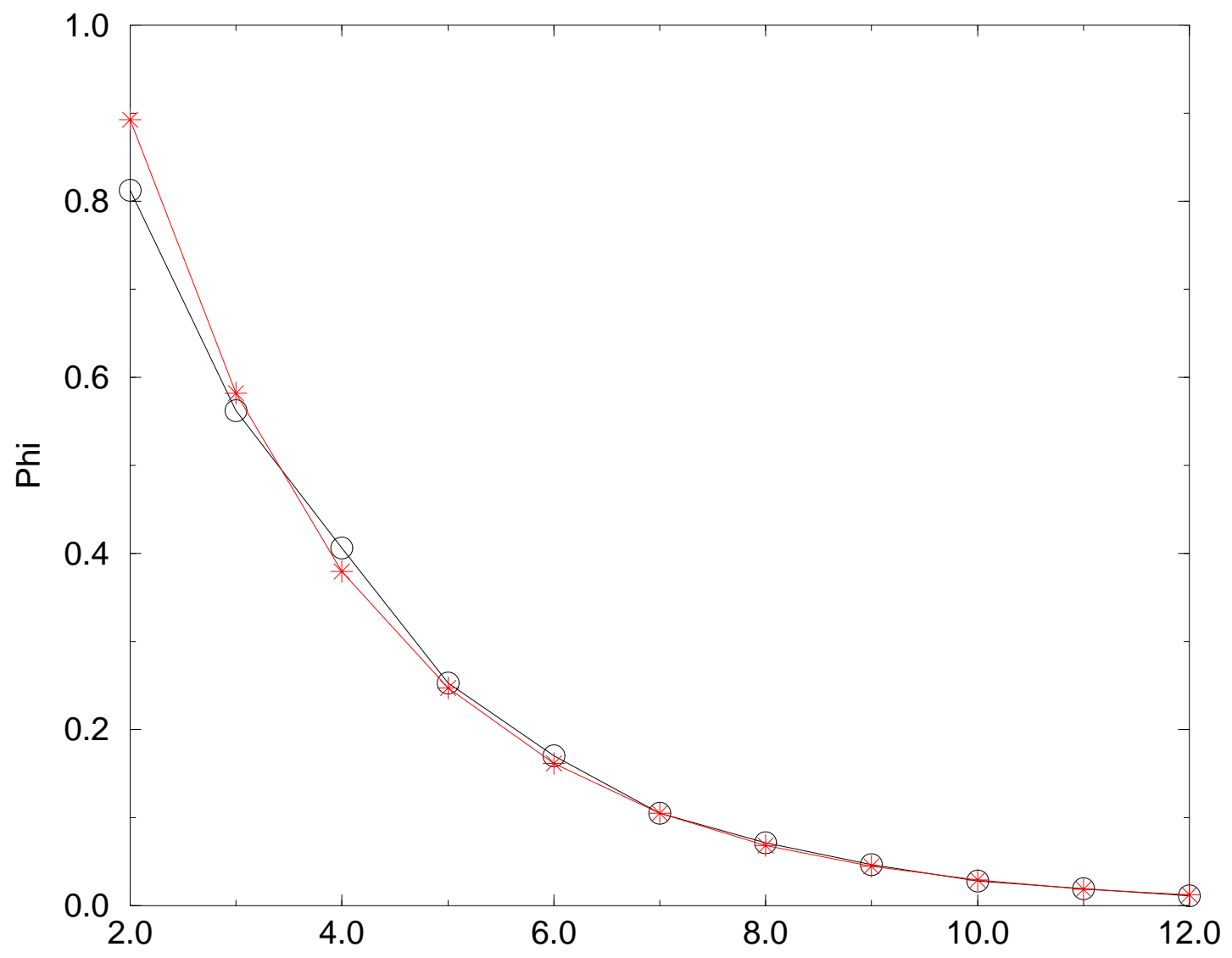

(a) 


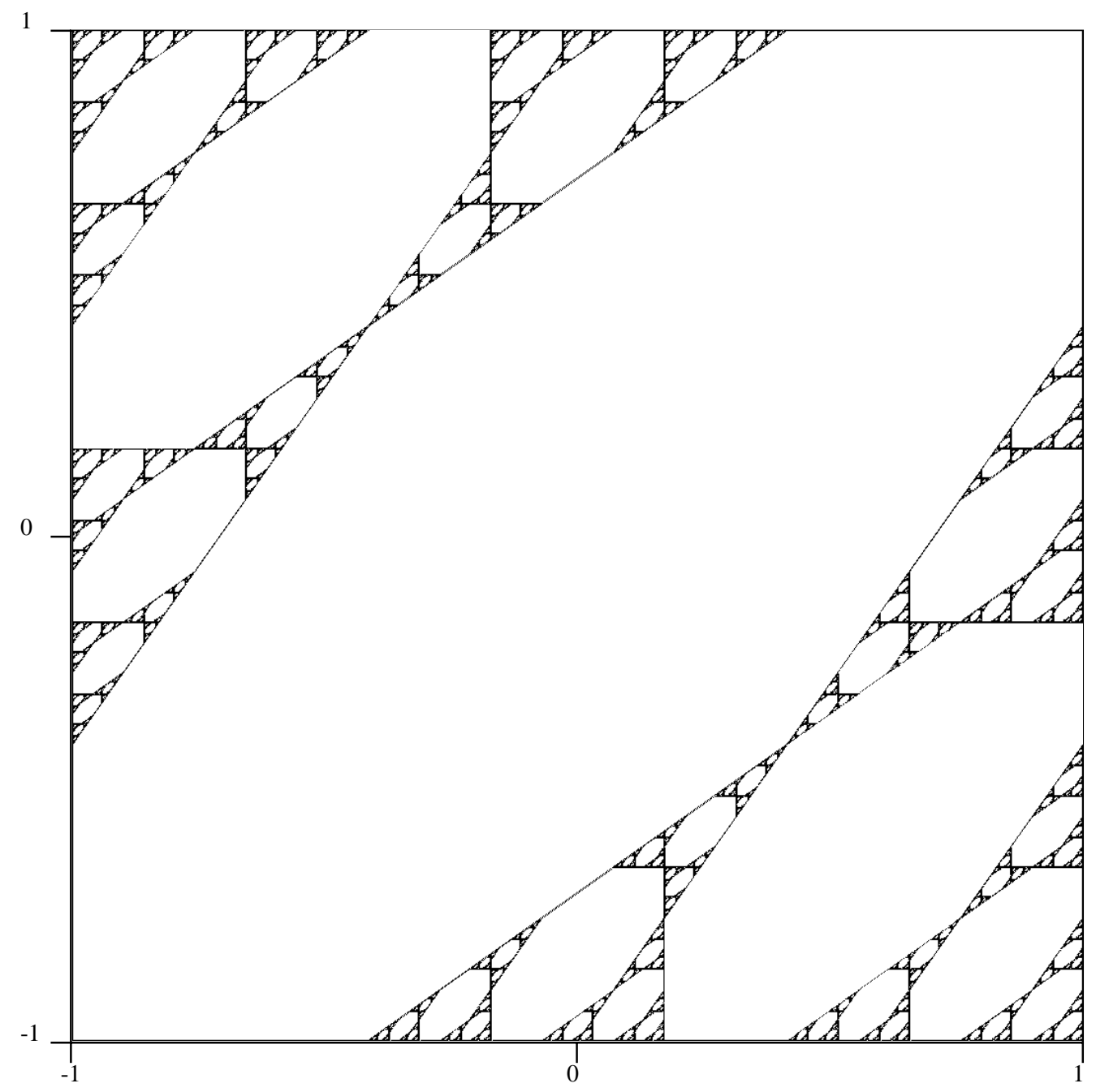

(b)

Figure 2: (a) the points marked by circles show $\Phi_{n}(\sqrt{2})$ as a function of $n=$ $2, \cdots, 12$. In this case there is a good fit to an exponential (shown by the stars) indicating that $\Phi(\sqrt{2})=0$. The exponential fit implies the set has a Minkowski dimension of 1.3835. (b) Approximation of $D$ for $n=10$. 


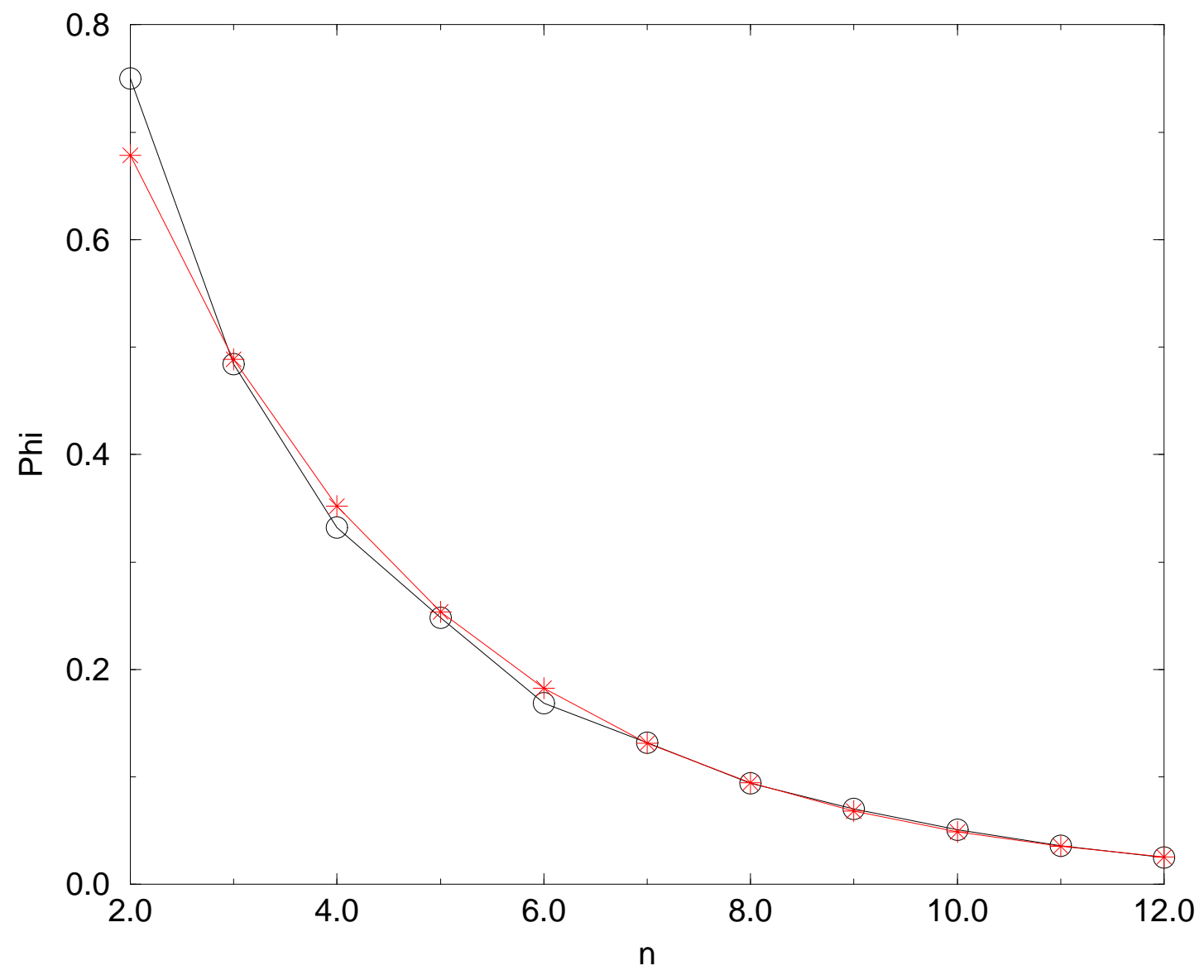

(a) 


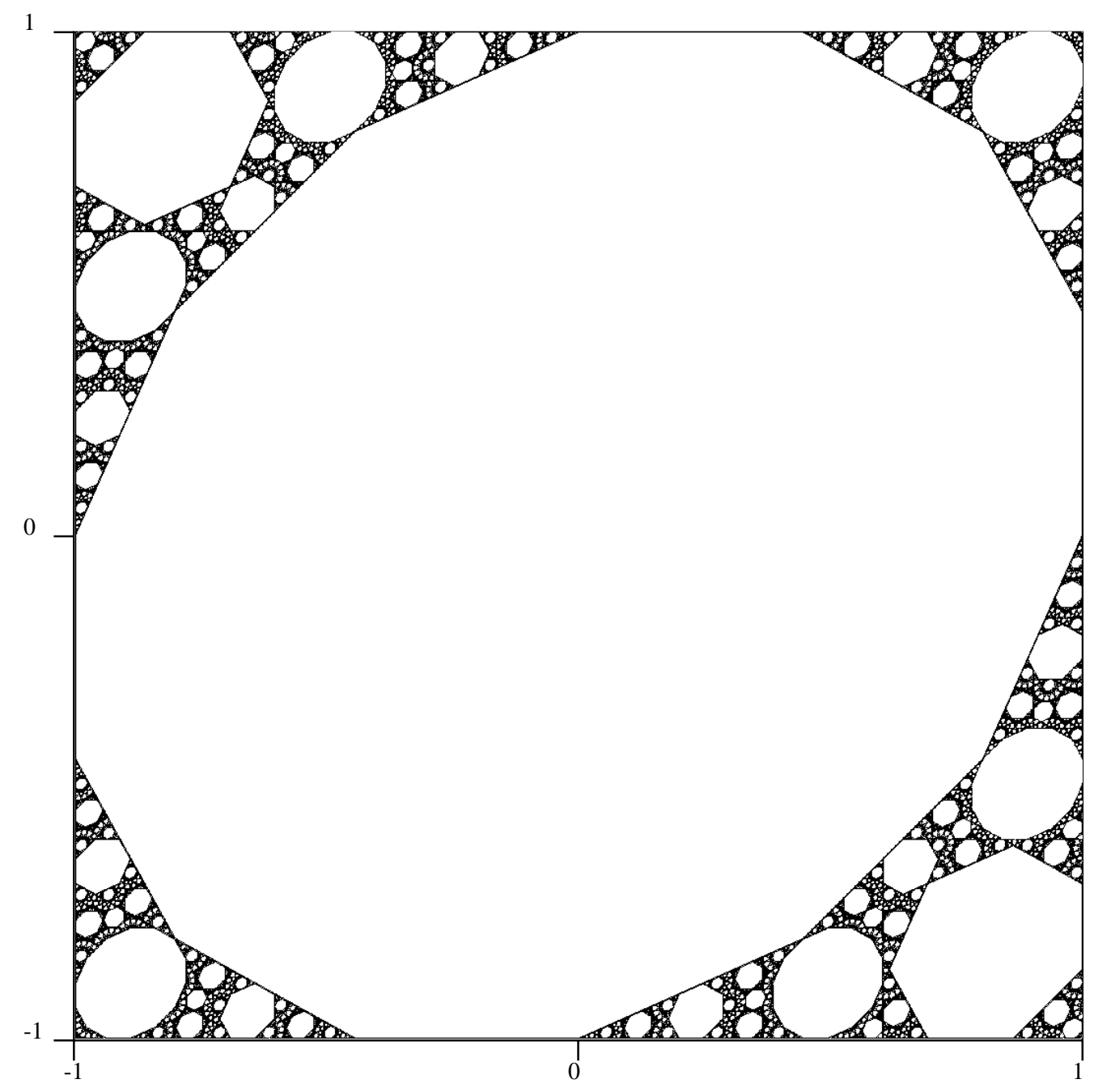

(b)

Figure 3: (a) the circles show $\Phi_{n}(0.44504)(0.44504=2 \cos 3 \pi / 7)$ as a function of $n=2, \cdots, 12$. In this case there is also a good fit to an exponential (shown by the stars) indicating that $\Phi(0.44504)=0$. The exponential fit implies a Minkowski dimension of 1.526. (b) Approximation of $D$ when $n=10$. 


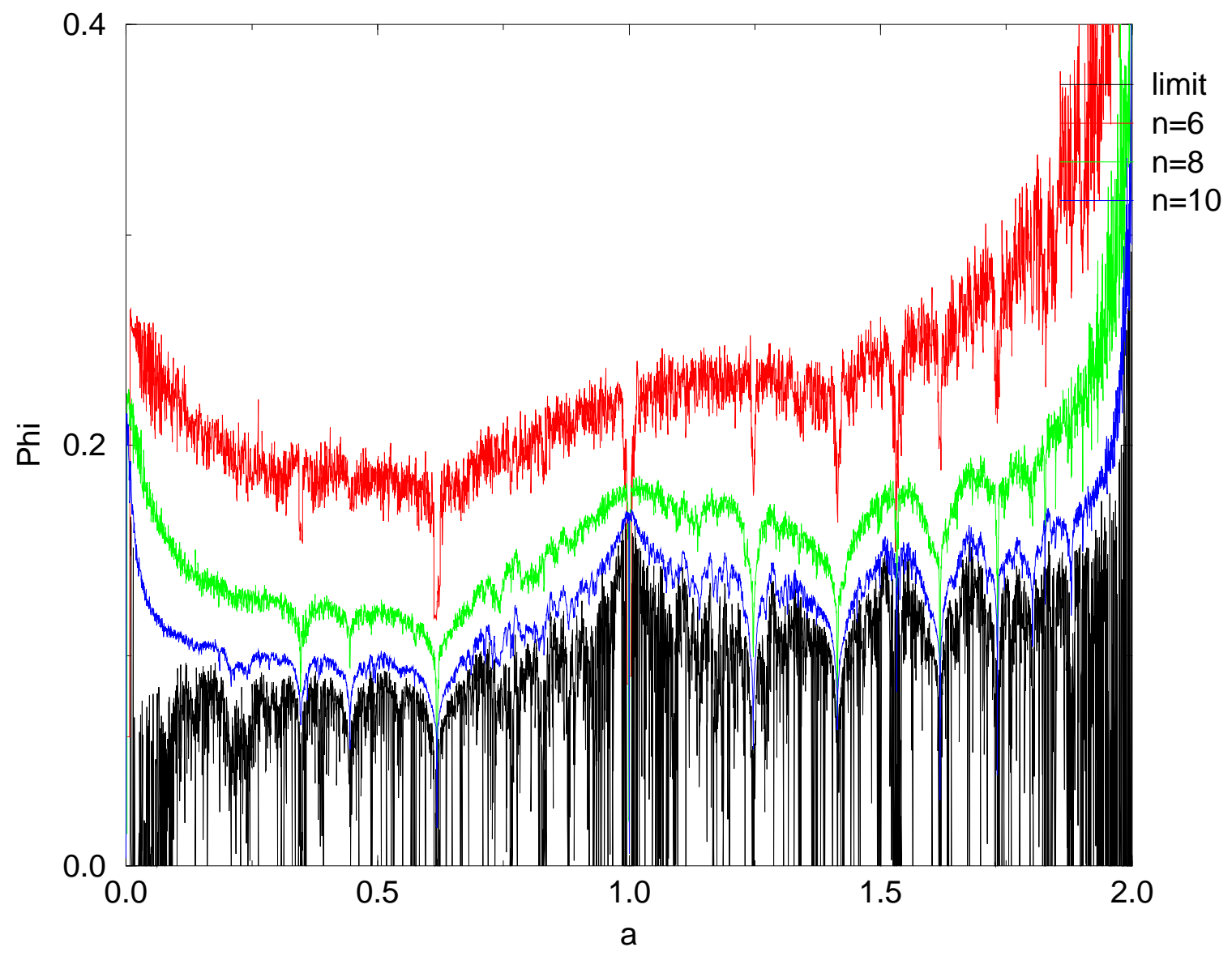

Figure 4: Graph of $\Phi_{n}(a)$ as a function of $a$ for $n=6,8$ and 10 and the limit $n=\infty$ extrapolated by fitting $A e^{n B}+C$ to these three values of $n$. Values were computed for $a=0.000012345+0.0005 k$ for $k=0 . .4000$. There is reasonable evidence that $\Phi(a)$ is non-zero for a large measure set of parameters. 


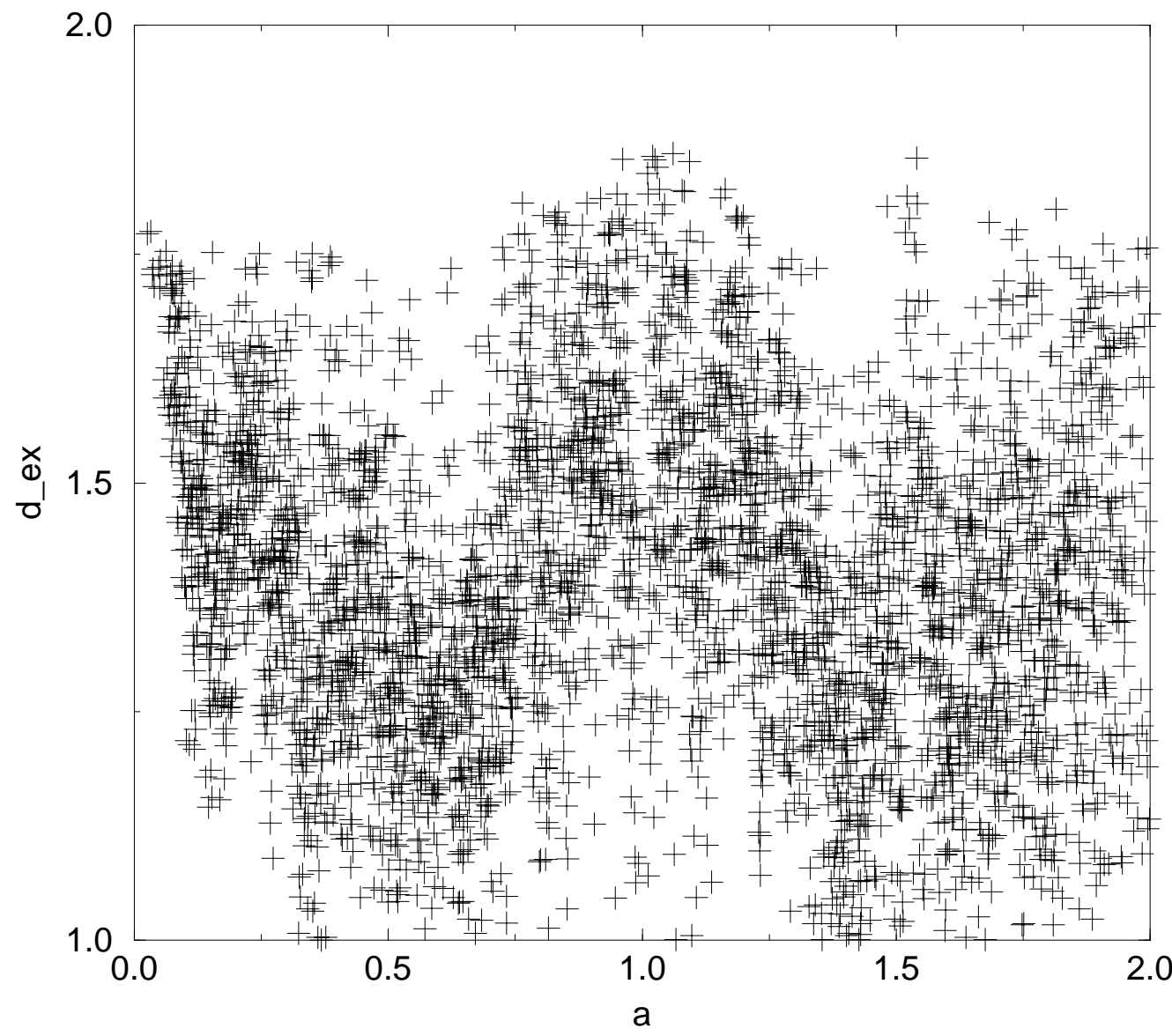

Figure 5: The exterior dimension (Minkowski dimension if $\Phi=0$ ) as a function of $a$ as calculated from the fit used for Figure 4; for the most part, the dimension is between 1 and 2 . 
Figure 6: The coloured regions show the areas of phase space taken up by some simple overflow sequences, from top to bottom starting with no overflow, period two overflow, period three overflow up to period 50 overflow. In the region $0<a<1$ the area of two additional islands with periods 6 and 10 overflow are shown. The curve below the coloured area is the approximation of $\Phi(a)$ obtained for $n=10$ shown in Figure 4 . The fact that this curve lies below the coloured area shows consistence of the two approaches. 


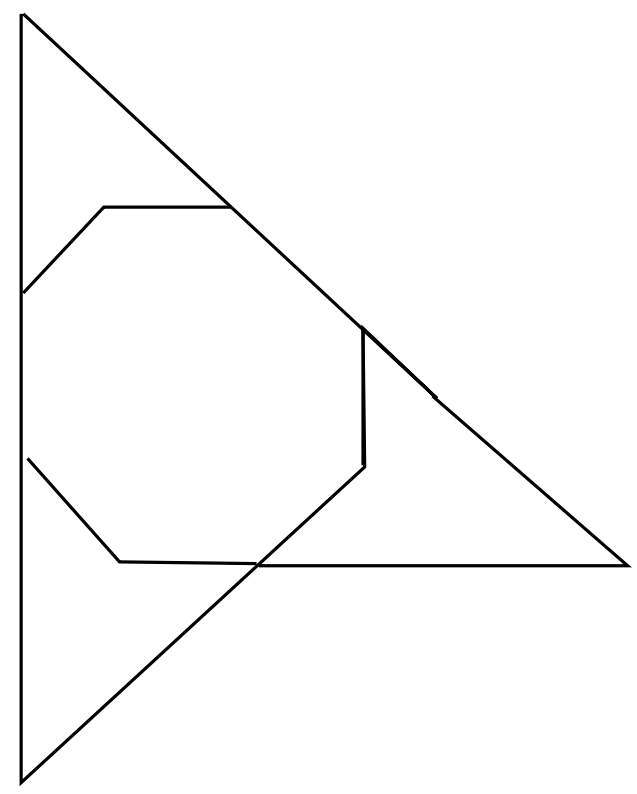

Figure 7: An approximation of $D$ for $a=\sqrt{2}$. This can be split into three self-similar patterns and so we can obtain the fractal (Hausdorff) dimension 1.37676. 


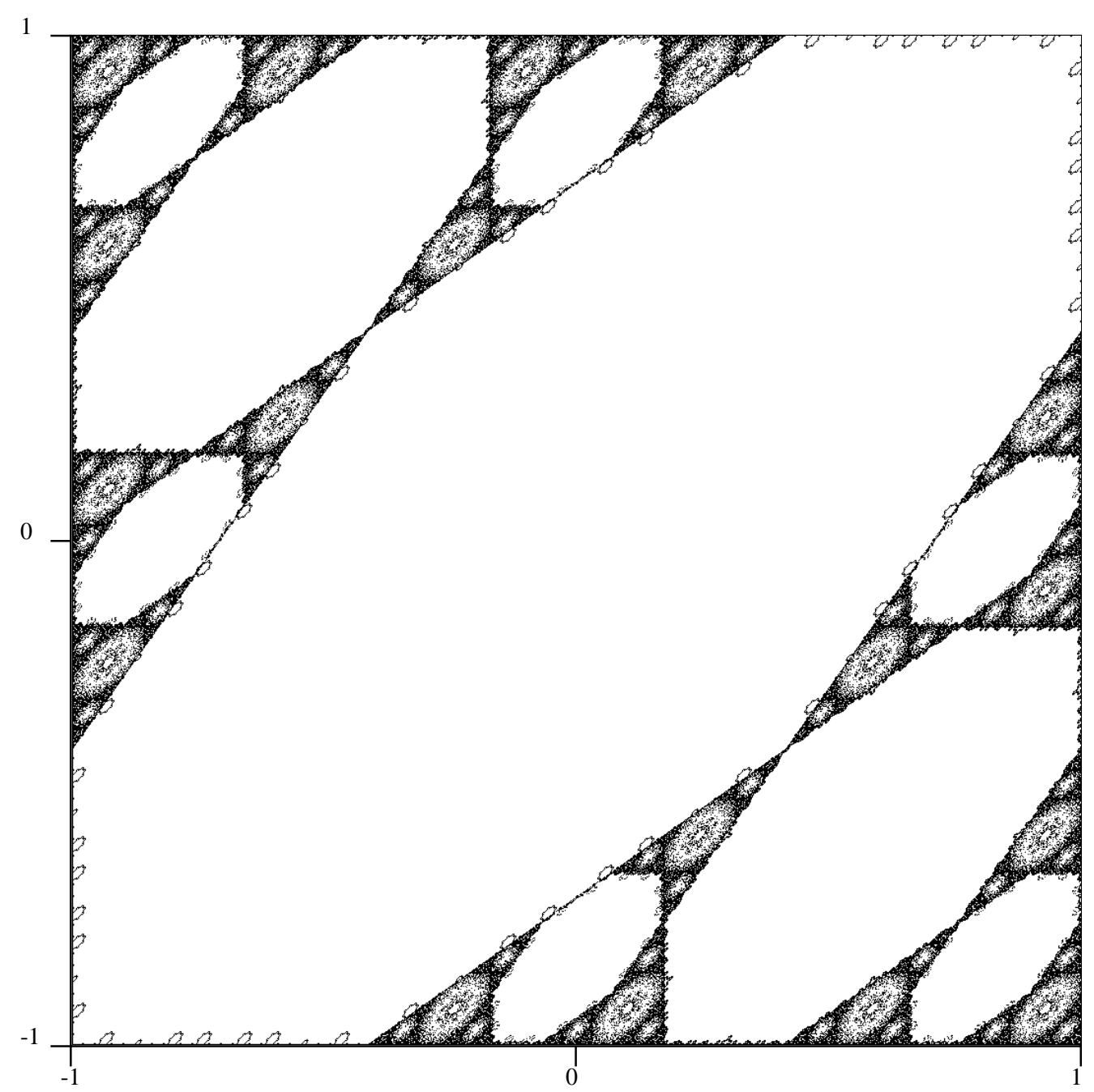

Figure 8: Approximation to $D$ for $a=\sqrt{2}$ calculated using finite precision approximation with $n=10$ (i.e. using 10-bit arithmetic). Compare with Figure 2 (b) which was calculated using double precision arithmetic. 


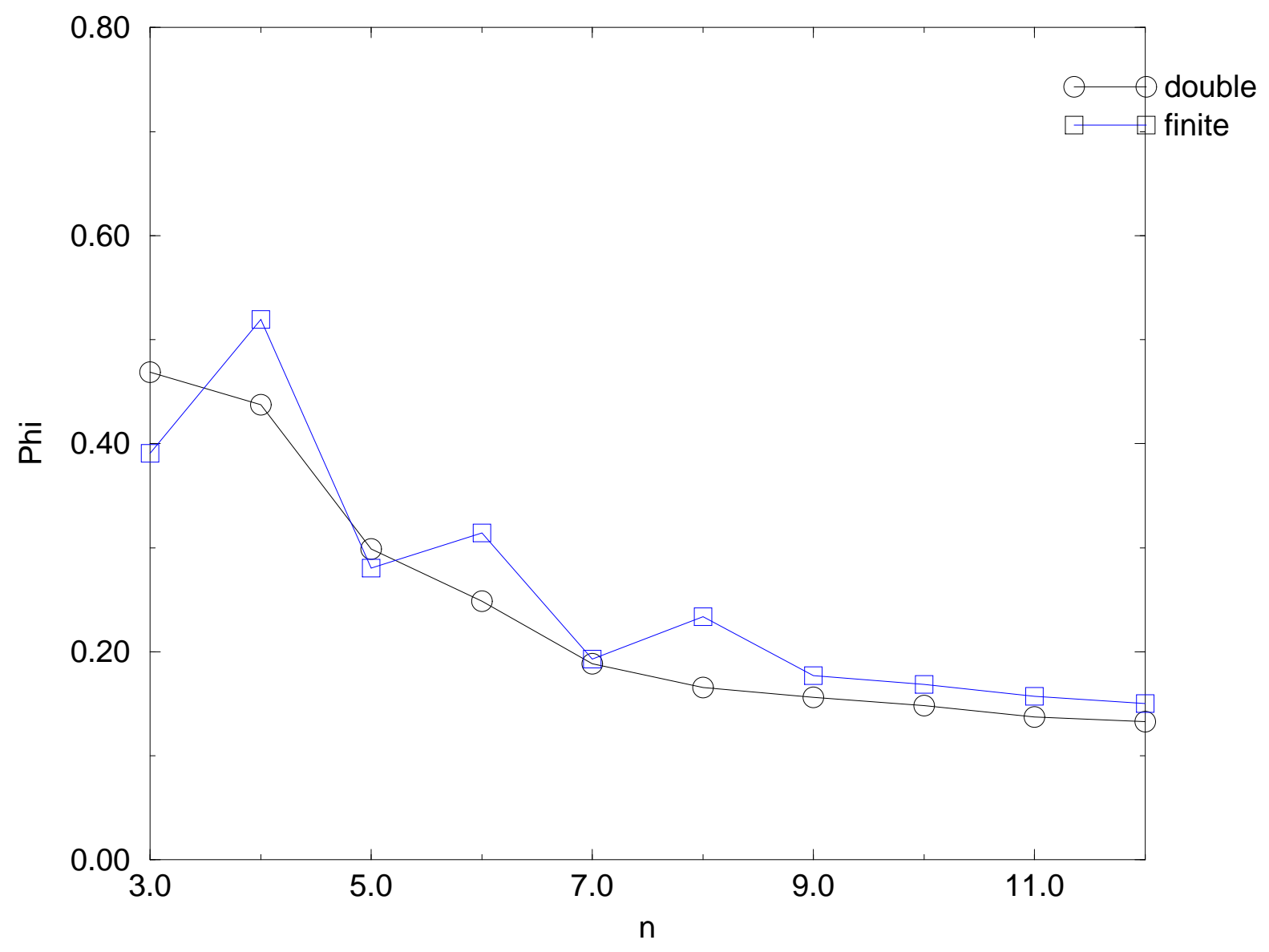

Figure 9: A comparison of approximations of $\Phi_{n}$ for $a=1.5$ and $n=3, \cdots, 12$ using double precision calculations (circles) and finite precision calculations with $n$-bit arithmetic (squares). Both curves appear to limit to a positive value, but the convergence of (b) is more irregular. 